\title{
Single-cell CRISPR screening in drug resistance
}

\author{
William Wang • Xiangdong Wang
}

Received: 13 March 2017 / Accepted: 25 April 2017 /Published online: 4 May 2017

C) Springer Science+Business Media Dordrecht 2017

Development of resistance and insensitivity to target therapy is a growing challenge during clinical treatment for patients with cancer that may require solutions via genome medicine (Gu \& Wang 2017). Gene changes such as mutations and heterogeneity could foster cancer evolution and reduce optimal efficacy of single or combinational therapies (Wang, 2016a). The current situation in clinical practice is that gene sequencing is performed to screen whether there are mutated genes which may be associated with the occurrence of drug resistance when target drugs fail to show effects in patients (Wu et al. 2017). More recently, gene mutation or heterogeneity are measured in tumor tissues harvested from cancer patients during surgery or biopsies, to select therapeutic strategies of target drugs and indicate the potential occurance of drug resistance. A number of obstacles limit efficient and precise detections of genes responsible for drug resistance during clinical therapy, e.g., intra- or inter-tumor heterogeneity between molecules, cells, and locations. Single-cell gene sequencing has been proposed as an alternative to detect intercellular heterogeneity of cancer (Qian et al., 2016; Wang 2015). Clustered regularly interspaced short palindromic repeats (CRISPR)/CRISPR associated (Cas9) is considered as an efficient and critical method and solution to modify expression and function of target genes for

W. Wang $\cdot$ X. Wang $(\bowtie)$

Zhongshan Hospital Institute of Clinical Science, Fudan University; Shanghai Institute of Clinical Bioinformatics, Shanghai, China

e-mail: xdwang@fuccb.com screening and evaluating target genes or to treat cancer (Fang \& Wang, 2016).

One of the most important functions is to in parallel measure the large-scale perturbations and phenotypes of individual cells by the integration of single-cell RNAseq with CROP-seq, CRISP-seq, Perturb-seq, and CRISPR/Cas9 as a single-cell CRISPR screen. Pooled CRISPR screening is a system where lentiviral transduction delivers the Cas9 nuclease and single guide RNAs (gRNA) into cells and knock out thousands of individual genes. As an important part of the system, the next-generation sequencing data from cells collected at the start of the screen are analyzed and compared with those at the end of a screen. Pooled CRISPR screening is an important tool and paradigm to identify function- and target-oriented genes in molecular characterization and mechanism of biological process, sensitivity and resistance against drugs, and screening of therapeutic strategies, rather than as a simple readout. The pooled CRISPR screening in mammalian cells was reported as a pooled, loss-of-function genetic screening approach and a powerful tool for systematic genetic analysis using a genome-scale lentiviral single gRNA library (Wang et al., 2014; Shalem et al., 2014). Such an approach can be used for tracking a complex mutant pool by massively parallel sequencing on the basis of the integration of single gRNA expression cassettes into the genome. Dixit et al. (Dixit et al., 2016a, b) developed Perturbseq integrating single-cell RNA-seq and CRISPR-based perturbations to detect complex transcriptional profiles at scale and understand gene function and regulation of transcription factors. This is a milestone study to 
evidence that Perturb-seq can be used to identify individual gene targets, signatures, and cell states affected by individual perturbations and their genetic interactions, and to deeply and accurately investigate effects of perturbations, their interactions, and diverse cell metadata, and pooled genomic functions. Adamson et al. (2016) combined Perturb-seq with CRISPR to detect unfolded protein responses and screen genomescale CRISPR interferences by measuring the number of perturbation and complexity of phenotypes.

Pooled CRISPR screening can be used to explore the regulatory mechanisms of cell responses to challenges. Datlinger et al. (2017) developed a complementary single-cell CRISPR screen to combine pooled CRISPR screening with single-cell RNA sequencing into a more widely and powerful application by directly linking gRNA expression to transcriptome responses in individual cells, according to the principle that gRNA and their induced biological functions exist within single cells. High-throughput comprehensive gene functions of complex regulatory mechanisms and heterogeneous cell populations can be promptly monitored by CRISPR droplet sequencing (CROP-seq) which enables pooled CRISPR screens with single-cell transcriptome resolution. Pooled screens can detect gRNA changes, transcriptome profiling, and droplet-based single-cell RNAseq profiles in each analysis identifies pathway signature genes and quantifies the effect of specific gRNAs on signatures. The CROP-seq lentiviral construct and CROPseq-Guide-Puro can be used for further validation of gRNAs. Parnas et al. (2015) presented a comprehensive study on the innate immune response of bone marrow derived dendritic cells from Cas9-expressing transgenic mice using the pooled CRISPR strategy. This is a new approach to explore the molecular mechanisms of dendritic cells with a pooled, genome-wide library of lentiviruses which express gRNAs and used to knockout genes in cells. Single gRNA abundance in dendritic cells with or without TNF $\alpha$ induced by lipopolysaccharide were measured by deep sequencing, in order to identify new regulators and functional modules of regulators with distinct regulatory effects. Pooled CRISPR screening is proposed as an opening of functional genomics research where a protein (Cas9) can be targeted to an exact genomic locus, through designing a specific short complementary nucleotide sequence with a common scaffold sequence, to constitute the gRNA bridging the protein and the DNA (Agrotis \& Ketteler, 2015). It is also the time to consider how to apply CRISPR-based methods in high-throughput functional genomics screening for drug discovery and development, resistance, and toxicology.

Pooled CRISPR screening is also a powerful tool to promote therapeutic genome editing and the functional interrogation of non-coding elements by mapping target-dependent phenotypes and perturbations. For example, enhancers as distal genetic elements positively regulate gene expression in an orientation-independent manner during gene expression. The enhancer map and screening can describe therapeutic genome editing and functional interrogation of non-coding genomic elements which bear an enhancer signature (Andersson et al., 2014). Canver et al. (2015) developed pooled CRISPR-Cas9 gRNA libraries to perform in situ saturating mutagenesis of human and mouse enhancers, on the basis of their understanding of an erythroid enhancer of BCL11A. Such screening can clearly demonstrate critical features and functions of enhancers, correlated with specific chromatin features.

DeJesus et al. (2016) applied the pooled CRISPR screening for novel regulators of SQSTM1 with genome editing by CRISPR-Cas9 on single gRNAs to target the endonuclease enzyme Cas9. CRISPR had advantages in the identification of SQSTM1 regulators by screening libraries of single gRNA as compared with pooled RNAi (Eng et al., 2016). Pooled CRISPR screening can be used to identify several novel regulators of SQSTM1 in the mechanism of SQSTM1 accumulation upon impaired autophagy-independent ufmylation. This is an example of pooled CRISPR screening as a powerful genetic tool for interrogating complex signaling networks and for identifying cellular targets.

Pooled CRISPR screening can be applied for identifying host factors during the responses to viral and bacterial infection or chemicals. Marceau et al. (2016) applied pooled CRISPR screening to undertake a comprehensive study on the mapping of host factors required for two Flaviviridae members and found endoplasmic reticulum-associated multi-protein complexes involved in signal sequence recognition, N-linked glycosylation, and degradation. Novel host targets in the oligosaccharyltransferase complex, endoplasmic reticulum-associated protein complexes, or flavin adenine dinucleotide were found to play critical roles in virus replication and the control of host sensitivity to infection. Gene changes, mutations, and heterogeneity play an important role in molecular mechanisms by which drug resistance occurs and drugs may target 
biological processes as a critical part of molecular toxicity (Chu et al., 2017; Gu \& Wang, 2016). Pooled CRISPR screens were used to investigate resistance to drug treatments, where gene changes and mutations at a resistance-inducing locus lead to the accumulation of mutagenized cells. The accuracy of specific gRNAs from the initial starting population to identify genes influences the sensitivity of screening, since gene changes and mutations may cause different sensitivities and phenotypes of cells to drugs. The complexity, interaction, and multiple losses of individual guides or genes can shadow the sensitivity of screening. However, multigenic phenotypes and diseases depend upon combinatorial gene functions and interactions, which requires high throughput CRISPR-Cas9-based screening with combinatorial sets of gRNAs. Wong et al. (2016) developed a new strategy to simply and efficiently make barcoded combinatorial gRNA libraries using combinatorial genetics en masse (CombiGEM)-based DNA assembly and termed CombiGEM-CRISPR. From the reality of disease pathogenesis and complexity of responses to drug, CombiGEM-CRISPR can be a better alternative for undertaking the systematic analysis of combinatorial gene functions and be more helpful in identifying and validating new gene and drug combinations responsible for the initiation of complex biological phenotypes and human diseases. By delivering libraries into human cells to create genetically ultradiverse cell populations harboring unique gRNA combinations, they can be tracked via barcode sequencing in pooled assays. Such strategies could result in new methods of targeted drug combinations for clinical applications. Wong et al. (2016) created a library of 23,409 barcoded dual gRNA combinations, and both identified and validated functions of gene pairs inhibiting ovarian cancer cell growth, using this strategy integrated with CRISPR-Cas-based knockouts and RNA-interference-based knockdowns.

Pooled CRISPR screening could also be a new alternative to better understand molecular mechanisms of drug side-effects, toxicity and toxicology. It was realized that drugs could change DNA repair mechanisms and cause the occurrence of gene mutations as an important part of toxicity three decades ago (Belitsky et al., 1985; Steinheider et al., 1986; Sinha et al., 1985). With the development of genetic technology, hereditary gene changes, mutations, and heterogeneity of human cells can result in the high susceptibility and resistance against drugs (Devine \& Patani, 2017; Wang, 2016b;
Wang, 2016c). Single-cell pooled CRISPR screening can play important role in understanding the mechanisms of genotoxicity and drug resistance. Single-cell RNA sequencing with pooled CRISPR screens demonstrated that the genomic perturbation and transcriptome were profiled in the same cell to illuminate the function of multiple factors and their interactions (Jaitin et al., 2016). It is possible that CRISP-seq can be applied to probe regulatory circuits of host genes responsible for responses to drugs, after which interactions and redundancies between drugs and genes using perturbed cells in in vitro and in vivo models. Dixit et al. (2016a, b) pooled single-cell RNA sequencing and CRISPR-based perturbations and found transcription factors regulating the response of dendritic cells to lipopolysaccharides. Such perturbation-sequencing demonstrated gene targets and signatures and cell states affected by individual perturbations and their genetic interactions. It indicates that new functions for regulators of differentiation, antiviral response, and mitochondrial function can be used to explore molecular mechanisms of genotoxicity and resistance against drugs.

In conclusion, gene changes, mutations, and heterogeneity play important roles in the development of drug genotoxicity and resistance. Single-cell CRISPR screen can be used to simultaneously measure the perturbation and phenotype of a cell by the integration of single-cell RNA-seq with CROP-seq, CRISP-seq, Perturb-seq, with CRISPR/Cas9. Pooled CRISPR screening can be used to explore regulatory mechanisms of cell responses to challenges, promote therapeutic genome editing and functional interrogation of non-coding elements, identify host factors during the responses to viral and bacterial infection or chemicals, and better understand molecular mechanisms of drug side-effect, toxicity, and toxicology.

\section{References}

Adamson B, Norman TM, Jost M, Cho MY, Nuñez JK, Chen Y, et al. A multiplexed single-cell CRISPR screening platform enables systematic dissection of the unfolded protein response. Cell. 2016;167(7):1867-1882.e21. doi:10.1016/j. cell.2016.11.048.

Agrotis A, Ketteler R. A new age in functional genomics using CRISPR/Cas9 in arrayed library screening. Front Genet. 2015;6:300.

Andersson R, Gebhard C, Miguel-Escalada I, Hoof I, Bornholdt J, Boyd M, et al. An atlas of active enhancers across human cell types and tissues. Nature. 2014;507(7493):455-61. 
Belitsky GA, Khovanova EM, Budunova IV, Sharuptis HG. Mycotoxin induction of somatic mosaicism in Drosophila and DNA repair in mammalian liver cell cultures. Cell Biol Toxicol. 1985;1(3):133-43.

Canver MC, Smith EC, Sher F, Pinello L, Sanjana NE, Shalem O, et al. BCL11A enhancer dissection by Cas9-mediated in situ saturating mutagenesis. Nature. 2015;527(7577):192-7.

Chu MP, Kriangkum J, Venner CP, Sandhu I, Hewitt J, Belch AR, Pilarski LM. Addressing heterogeneity of individual blood cancers: the need for single cell analysis. Cell Biol Toxicol. 2017;33(2):83-97.

Datlinger P, Rendeiro AF, Schmidl C, Krausgruber T, Traxler P, Klughammer J, et al. Pooled CRISPR screening with singlecell transcriptome readout. Nat Methods. 2017;14(3):297-301.

DeJesus R, Moretti F, McAllister G, Wang Z, Bergman P, Liu S, et al. Functional CRISPR screening identifies the ufmylation pathway as a regulator of SQSTM1/p62. elife. 2016;5: e17290.

Devine H, Patani R. The translational potential of human induced pluripotent stem cells for clinical neurology: the translational potential of hiPSCs in neurology. Cell Biol Toxicol. 2017;33(2):129-44.

Dixit A, Parnas O, Li B, Chen J, Fulco CP, Jerby-Arnon L, et al. Perturb-Seq: dissecting molecular circuits with scalable single-cell RNA profiling of pooled genetic screens. Cell. 2016a;167(7):1853-66.

Dixit A, Parnas O, Li B, Chen J, Fulco CP, Jerby-Arnon L, et al. Perturb-Seq: dissecting molecular circuits with scalable single-cell RNA profiling of pooled genetic screens. Cell. 2016b;167(7):1853-1866.e17. doi:10.1016/j. cell.2016.11.038.

Eng CH, Wang Z, Tkach D, Toral-Barza L, Ugwonali S, Liu S, et al. Macroautophagy is dispensable for growth of KRAS mutant tumors and chloroquine efficacy. Proc Nat Acad Sci USA. 2016;113:182-7.

Fang H, Wang W. Could CRISPR be the solution for gene editing's Gordian knot? Cell Biol Toxicol. 2016;32(6):465-7.

Gu J, Wang X. New future of cell biology and toxicology: thinking deeper. Cell Biol Toxicol. 2016;32(1):1-3.

Gu J, Wang X. Piercing the "armor" of lung cancer with genome medicine. Semin Cancer Biol. 2017;42:1-3.

Jaitin DA, Weiner A, Yofe I, Lara-Astiaso D, Keren-Shaul H, David E. Dissecting immune circuits by linking CRISPRpooled screens with single-cell RNA-Seq. Cell. 2016;167(7): 1883-1896.e15.
Marceau CD, Puschnik AS, Majzoub K, Ooi YS, Brewer SM, Fuchs G, et al. Genetic dissection of Flaviviridae host factors through genome-scale CRISPR screens. Nature. 2016;535(7610):159-63.

Parnas O, Jovanovic M, Eisenhaure TM, Herbst RH, Dixit A, Ye CJ. A genome-wide CRISPR screen in primary immune cells to dissect regulatory networks. Cell. 2015;162(3):675-86.

Qian M, Wang DC, Chen H, Cheng Y. Detection of single cell heterogeneity in cancer. Semin Cell Dev Biol. 2016; doi:10.1016/j.semcdb.2016.09.003.

Shalem O, Sanjana NE, Hartenian E, Shi X, Scott DA, Mikkelsen TS, et al. Genome-scale CRISPR-Cas9 knockout screening in human cells. Science. 2014;343(6166):84-7.

Sinha AK, Linscombe VA, Gollapudi BB, Jersey GC, Park CN. Analysis of sister chromatid exchanges in lymphocytes cultured from 71 healthy men. Cell Biol Toxicol. 1985;1(4): 333-42.

Steinheider G, Neth R, Marquardt H. Evaluation of nongenotoxic and genotoxic factors modulating the frequency of micronucleated erythrocytes in the peripheral blood of mice. Cell Biol Toxicol. 1986;2(1):197-211.

Wang X. Single cell sequencing and systems immunology. Serial Books: Translational Bioinformatics; Ed: Wang XD, Volume 5, Springer Netherlands; 2015. doi: 10.1007/978-94-0179753-5.

Wang X. Gene mutation-based and specific therapies in precision medicine. J Cell Mol Med. 2016a;20(4):577-80.

Wang X. New biomarkers and therapeutics can be discovered during COPD-lung cancer transition. Cell Biol Toxicol. 2016b;32(5):359-61.

Wang X. CBT profiles of cabozantinib approved for advanced renal cell carcinomas. Cell Biol Toxicol. 2016c;32(4): 259-61.

Wang T, Wei JY, Sabatini DM, Lander ES. Genetic screens in human cells using the CRISPR/Cas9 system. Science. 2014;343(6166):80-4.

Wong AS, Choi GC, Cui CH, Pregernig G, Milani P, Adam M, et al. Multiplexed barcoded CRISPR-Cas9 screening enabled by CombiGEM. Proc Natl Acad Sci U S A. 2016;113(9): 2544-9.

Wu D, Wang DC, Cheng Y, Qian M, Zhang M, Shen Q, Wang X. Roles of tumor heterogeneity in the development of drug resistance: a call for precision therapy. Semin Cancer Biol. 2017;42:13-9. 